\title{
Quantitative Ultrasound Elastography of Breast: A Review and Update with Emphasis on Shear Wave Imaging (ARFI)
}

\author{
Duzgun Yildirim ${ }^{*}$, Özlem Akıncı², Deniz Esin Tekcan ${ }^{3}$ \\ ${ }^{1}$ Department of Medical Imaging, Acibadem University, Vocational School of Health Sciences, Istanbul, Turkey \\ ${ }^{2}$ Department of Radiology, Sancaktepe Prof. Dr. Ilhan Varank Training and Research Hospital, Istanbul, Turkey \\ ${ }^{3}$ Department of Radiology, Acibadem University, Istanbul, Turkey \\ Email: ${ }^{\star d r d u z g u n y i l d i r i m @ g m a i l . c o m ~}$
}

How to cite this paper: Yildirim, D., Akıncı, Ö. andTekcan, D.E. (2021) Quantitative Ultrasound Elastography of Breast: A Review and Update with Emphasis on Shear Wave Imaging (ARFI). Open Journal of Medical Imaging, 11, 58-72.

https://doi.org/10.4236/ojmi.2021.112006

Received: April 25, 2021

Accepted: June 27, 2021

Published: June 30, 2021

Copyright $\odot 2021$ by author(s) and Scientific Research Publishing Inc. This work is licensed under the Creative Commons Attribution-NonCommercial International License (CC BY-NC 4.0). http://creativecommons.org/licenses/by-nc/4.0/

\begin{abstract}
Elastography is a method which determines the stiffness of tissues with the help of ultrasound technology and exhibits more quantitative data according to palpation that made during physical examination. Elastography has relatively entered to routine use in the breast evaluation with imaging techniques. Although palpation has a very long history, elastography has been used since 1990s. Elastography is used as an adjunct to conventional gray scale ultrasound and can identify the stiffness of the tissues non-invasively. Today, it is used as two separate technological modalities: strain elastography and shear wave elastography. The aims of these modalities are to increase the sensitivity of the separation between malignant and benign lesions, reduce the unnecessary biopsy processes and to provide a more accurate Breast Imaging Reporting and Data System (BIRADS) categorization of the breast lesions. In this article, we aimed to review the clinical utilization and benefits of elastography in differantial diagnosis of breast lesions, BIRADS categorization, and biopsy decision making in the light of current literature.
\end{abstract}

\section{Keywords}

Breast, Elastography, Shear Wave Elastography, Ultrasound

\section{Introduction}

Mammography and ultrasound are the main methods used together in breast cancer screening. However, both methods present some limitations. Mammography performed in dense breasts may often yield false-negative results. US is sensitive in the detection of lesions, but specificity is poor as most solid lesions 
are benign [1]. Magnetic Resonance Imaging (MRI) can also be used for differentiation of benign and malignant lesions of the breast. According to several studies, MRI has a high sensitivity $(89 \%-100 \%)$, but a variable specificity $(37 \%$ 97\%), for the detection of breast cancer [2]. Also, as different application, Doppler ultrasonography was failed to provide the expected results in breast cancer diagnosis or differentiation. Although increased vascularity with Doppler ultrasonography increases the likelihood of malignancy, it may increase unnecessary biopsy rates when not evaluated together with other criteria in the BIRADS ultrasonography lexicon.

Any procedure in addition to ultrasonography (US) such as requiring contrast (as in MRI) and X-ray exposure (especially for repeated mammographies) or invasive procedures (as for biopsy) increases the cost. This means that the main factor in the sonographic characterization of breast lesions is the gray-scale morphological features of the lesion [3] [4].

Ultrasonography still could not reach the desired level in differentiation of malignant and benign lesions, even though, the increase in frequency up to 18 20 megaHertz in gray-scale, large matrix transducers and addition of new softwares as tissue harmonic imaging (THI) and spatial compound imaging (SCI). For example, increasing the effectiveness of ultrasound with SCI reduces the degree of posterior acoustic shadowing [5]. Power doppler ultrasound with the vibration generated by the operator assist to some cases, but it still may not be very effective, especially in small lesions [6].

At this point, the introduction of elastograpy has become the savior for radiology for the purpose of re-evaluation of the indetermined breast lesions detected by the other modalities and for a second look assessment [7]. Certainly, after gaining the necessary experience and completing the learning curve initially for strain elastography and subsequently for shear wave elastography; it will be easier to determine the benign and the malignant breast lesions. Although elastography is easy to perform, training and technical knowledge are required in order to obtain images permitting a correct interpretation. This method is an extremely operator dependent system as reported before in previous studies [1]. In the recently developed strain elastography softwares, color indices of the lesions are quantified mathematically minimize the impact of the operator at this stage [8] [9].

Today, strain elastography technique has replaced with shear wave elastography (SWE) in elastographic investigations; and is likely to remain as a supportive semi-quantitative modality for generally superficial lesions or hard lesions where the posterior contours of the lesions cannot be evaluated at all. The use of SWE as an adjunct to conventional B-mode ultrasound can increase diagnostic confidence and improve patient management. More specifically, SWE features can help reclassify BI-RADS category 3 or 4 a lesions by morphologic criteria on conventional B-mode ultrasound [10].

In this study, we aimed to evaluate the new and advanced imaging technology SWE in breast and axillary lesions. With the help of these images and data 
through the literature, we also aimed to specify all the features of this technique. In addition, with this review, we attempted to explain the expected developments associated with SWE and what should a clinician or a surgeon wait from these findings obtained using this technique.

\section{Ultrasound Elastography Techniques}

Tissues in the breast with different contents give a different response to deformation by compression and that is called elasticity [11] [12]. US elastography is an imaging technique that can be used to assess the stiffness or elasticity of breast masses, which is analogous to clinical palpation with US for a mass. The distinction between clinical palpation and elastography is that the former allows only a subjective judgment of the stiffness of a lesion, while elastography assesses tissue-specific differences in stiffness and/or elasticity, as lesions with an abnormal internal structure have altered elasticity [12] [13].

In Ultrasound elastography techniques (USE), tissue stiffness (elasticity) could be measured after applying an external force to the tissue surface [14] [15]. The first technique is called as real time strain elastography.

Since the first launch, plenty of studies were shared in literature and this method was converted to semi-quantitative modality from its first qualitative form [16] [17] [18]. In addition to new ratio measurements at obtained images, many other techniques (color index, pixel intensity etc) and the research process has recently gained improvements to be an operator independent modality [8] [19]. However, in its current mode, it is extremely operator dependent and not convenient in non-superficial lesions. It is available and still widely used as a semi-quantitative method on most of the renders' US machines for being less expensive [20].

In another elastography modality shear wave elastography (SWE), tissues are compressed with a special push pulse and the tissues are displaced at micron level. With that displacement, thorough the compressed tissues, shear waves are generated vertical to the course of push pulse way. So, this technology (acoustic radiation forced pulse imaging-virtual touch imaging; ARFI-VTI) quantifies stiffness (elasticity) without manual compression [21] [22]. ARFI that measures elasticity without the need for physical compression is an excellent replacement for strain imaging. Hard tissues can be compressed lesser and through these intense-cellular interfaces shear waves travel faster than soft ones with this technique [23].

Briefly, by these simple methods, specific images are obtained additional to conventional gray scale images. It could be possible to measure the stiffness of targetted area and it could be possible to define the tissue content indirectly with ROI measurements and specific color maps (velocity or quality maps). Generally, we will concentrate on the ARFI method using the most common one (Siemens, Erlangen, Germany), in this review. In principal, all software and hardware are based on a similar logic with minor differences (hard or soft tissue dif- 
ference in color representing, the unit reflects the stiffness is $\mathrm{m} / \mathrm{s}$ or $\mathrm{kPa}$, etc.).

\subsection{Aim of This Study}

We aim to determine the features of SWE in normal breast tissue, the parenchymal appearences, SWE characteristics of benign and malignant breast lesions, then to evaluate its usage in premalignant and non-mass lesions and lastly the effect on BIRADS classification. Then, with the support of literature data and in the light of updates we will point some special clues and conclude the role of SWE in breast lesions.

\subsection{Breast SWE with Related Images}

The ARFI method is a less operator dependent technique. Lesions may be compared with the adjacent normal tissues. Mild compression or just touching to the breast surface is needed, because also pre-compression of the tissue may amplify the shear wave velocity (SWVs) [24]. Therefore -compared to other available elastography techniques-measuring the shear wave propagation induced by the automated push pulse is meant to be the most standardized and examiner independent technique.

Kim et al found that the mean elasticity score $(3.7 \pm 1.0$ vs. $1.6 \pm 0.8, \mathrm{p}<0.01)$, SWV $(4.23 \pm 1.09 \mathrm{~m} / \mathrm{sec}$ vs. $2: 22 \pm 0.88 \mathrm{~m} / \mathrm{sec}, \mathrm{p}<0.01)$ and SR $(5.69 \pm 1.63$ vs. $2.69 \pm 1.40, \mathrm{p}<0.01$ ) were significantly higher for malignant lesions than benign lesions with this technique and especially if the ARFI is added, the differential diagnosis in breast lesions reached up to $93.6 \%$ [25]. The results for US combined with ARFI elastography and SR values were $97.5 \%$ sensitivity, $92.3 \%$ specificity, $93.6 \%$ accuracy, a $79.6 \%$ positive predictive value (PPV), and a $99.1 \%$ negative predictive value [25]. In another study, specificity was $62.5 \%$ among the BIRADS 4 breast lesions and $72.9 \%$ with addition of virtual touch imaging quantification (VTIQ) to the gray scale. VTIQ qualitative and quantitative elastography has the potential to further characterise B-mode detected breast lesions, increasing specificity and reducing the number of unnecessary biopsies [26].

Clinicopathological features such as histological type, histological grade, tumor size, the presence of lymph node metastasis, and lymphovascular invasion are known as prognostic factors of breast cancer. Regarding SWE, high nuclear and histologic grade, large invasive size and lymphovascular invasion were reported to be associated with increased stiffness of invasive breast cancer [27] [28] [29] [30]. Estrogen receptor negativity, progesterone receptor negativity, p53 positivity, and Ki-67 positivity were significantly associated with a higher E-ratio, and triple-negative and HER2-positive tumors showed greater stiffness than estrogen receptor-positive tumors [29] [30]. Interestingly, some aggressive tumors, such as high grade cancers and triple-negative tumors, are likely to be assessed as BI-RADS category 3 in B-mode ultrasound, but SWE may provide additional information for diagnosing those benign-looking malignancies [30].

In the literature, the cut-off values were also different to distinguish benign 
and malignant lesions according to type of device and different operators [25] [31]. The cut off value is up to $91 \%$ in malignant lesions with SWV exceeds probability and below the $10 \%-11 \%$ in non-malignant lesions according to recent meta-analysis result [32]. The cut-off value for malignancy was $2.55 \mathrm{~m} / \mathrm{s}$ level with point shear wave, it has partly elevated and reached to $3.59 \mathrm{~m} / \mathrm{s}$ level by the help of new technology named VTIQ which can make smaller measurements with ROI in 2-D form [33] [34] [35] [36] [37]. A quantitative data could be measured quantitatively with mild compression by the VTIQ method (Figure 1). In more recent systems, the resolution of B-mode gray scale images are sufficiently high and provides opportunity for quality control of obtained SWV maps by the help of VTIQ quality map [38].

In this modality, cysts have a typical "bull's eye" appearance (small size, white center, peripheral black circle). Therefore, hypoechoic or hypo-anechoic or complicated cysts, galactoceles with solid appearences can be clearly distinguished regardless of being simple or complicated (Figure 2).

For solid lesions, not only internal structures and peripheral contours, also the reaction in perilesional area can be clearly demonstrated and evaluated in detail, by SWE imaging. In this evaluation, while benign lesions are encoded as a more hyperechoic in gray scale displacement maps, and iso-elastic type colors with background parenchyma and fatty tissue or soft in VTIQ maps. In contrary, non-necrotic components of malignant lesions are encoded with more rigid

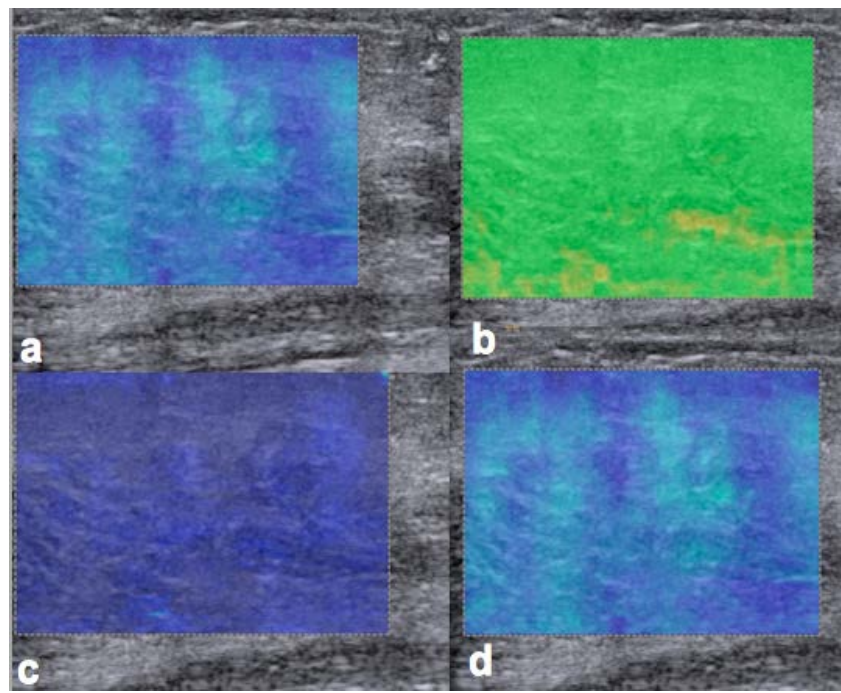

Figure 1. (a) Superposed on normal gray-scale US appearence of a normal parenchymal area, study with shear wave velocity (SWV) color map; (b) associated quality map appears. Color evaluation can be made from the bar on the side on these maps. Homogenous green color on quality map presents the homogeneous spread of the sound beam and indicates that the obtained map is more reliable. According to this map, we understand that we need to measure SWV from the more homogeneous regularly flor; (c)-(d) represent the SW time and coloured displacement maps, respectively. 


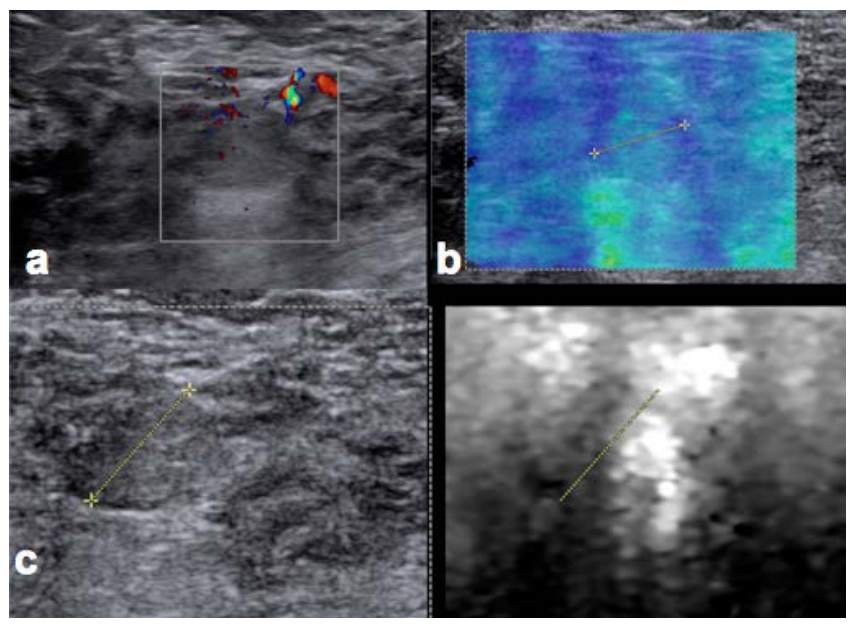

Figure 2. Image obtained from the 35-year-old case, 6 months after lactation ends; (a) echogenic on gray scale; (b) an echogenic centimetric mass, showing color scattering (artefactual) suggests questionable bleeding on Doppler window is monitored; (c) bulls eye view on gray scale acoustic radiation force imaging (ARFI) displacement (shining inside the hypoechogen ring), and (d) inside part green, outside with blue paint, soft and benign lesion image in virtual touch imaging quantification (VTIQ) window is consistent with dense cyst.

dense colors (In our cases, all the rigidity increases toward the red). Also, desmpolastic reaction created by malignant infiltration and ductal extension also could be defined by color or velocity maps as surrounding rigidity which extends beyond the gray scale tumoral margins (Figure 3). As well as these quantified measurements, semiquantitative assessment utilization such as color scale, ratio or index measurements in strain elastography has to be reduced gradually.

Fibrocystic changes and adenosis like hyperplastic changes that can create a premalign impression by mimicking the ductal carcinoma in situ (DCIS)'s usual appearance (Figure 4 and Figure 5). The separation of changes in the fibrocystic complex echo becomes important at this point. For these nonspecific lesions, when the lesional color pattern symbolizing the malignant degree of hardening than the surrounding normal breast tissue, BIRADS categorization will also be upgraded and rather than follow-up, planning for a biopsy will be more possible.

When a biopsy is required within the identified lesion area, the cyto-histopathological sample may be easily obtained with guidance of elastography, instead of using only gray scale images. This feature is not only typical for malignant like lesions, but also for suspicious natured BIRADS 3 lesions (Figure 6). Thus, planning of the medical or surgical treatment and complementary chemoradiotherapy can be performed from the beginning of the diagnostic stage of the disease. Fibrocystic changes starting from simple hyperplasia, adenosis, florid hyperplasia; continue with the flat epithelial atypia, atypical ductal hyperplasia and could terminate at least with a low-high grade DCIS spectrum. All these unfavorable course can be diagnosed with the help of the specific high 


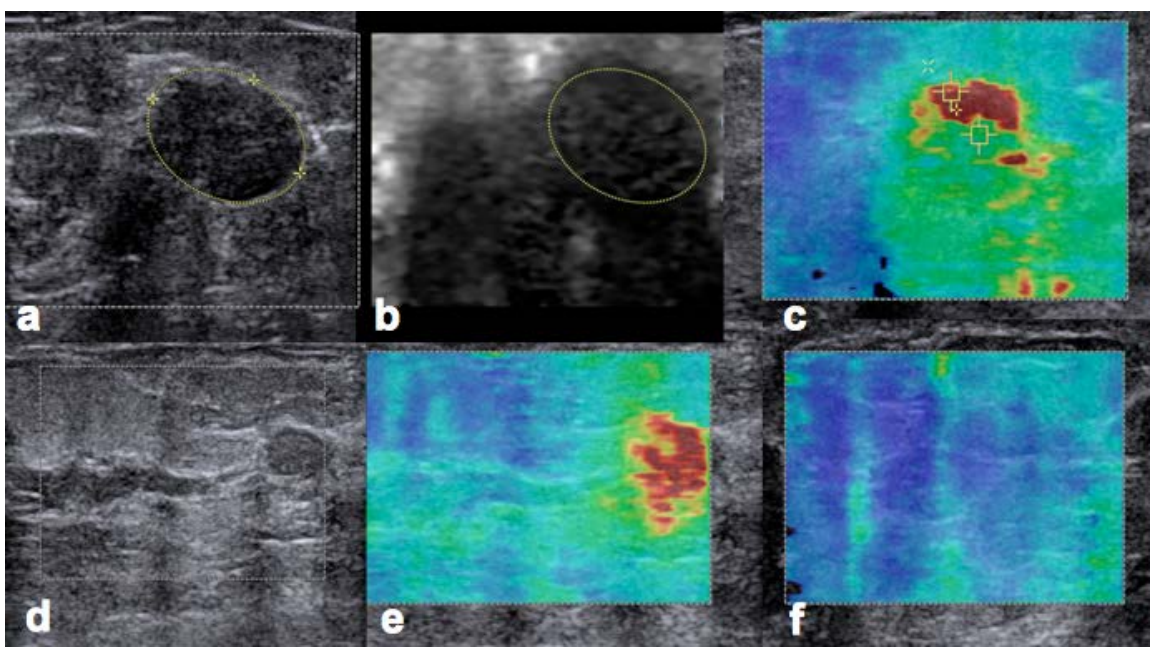

Figure 3. 47-year-old female patient. (a) Uniform limited, palpable, suspicious microlobulated hypoechogen lesion, normal gray scale contours of the lesion overlow; (b) a rigid desmoplastic ring is observed, on displacement map; (c) In VTIQ map, especially on peripheral non-necrotic ring, yellow-orange-red discolorations representing SWV increases in malignant spectrum are monitored; (d) blue weighted homogeneous color pattern in normal tissue; (e) the distorsion is converted to a darker green heterogeneous color, in traces found at malignant ductal extension; ( $f$ ) although this extension has no malignant appearence on gray scale image, VTIQ is illustrative for an expansion watched on gray scale that located deeply along a ductal trace.

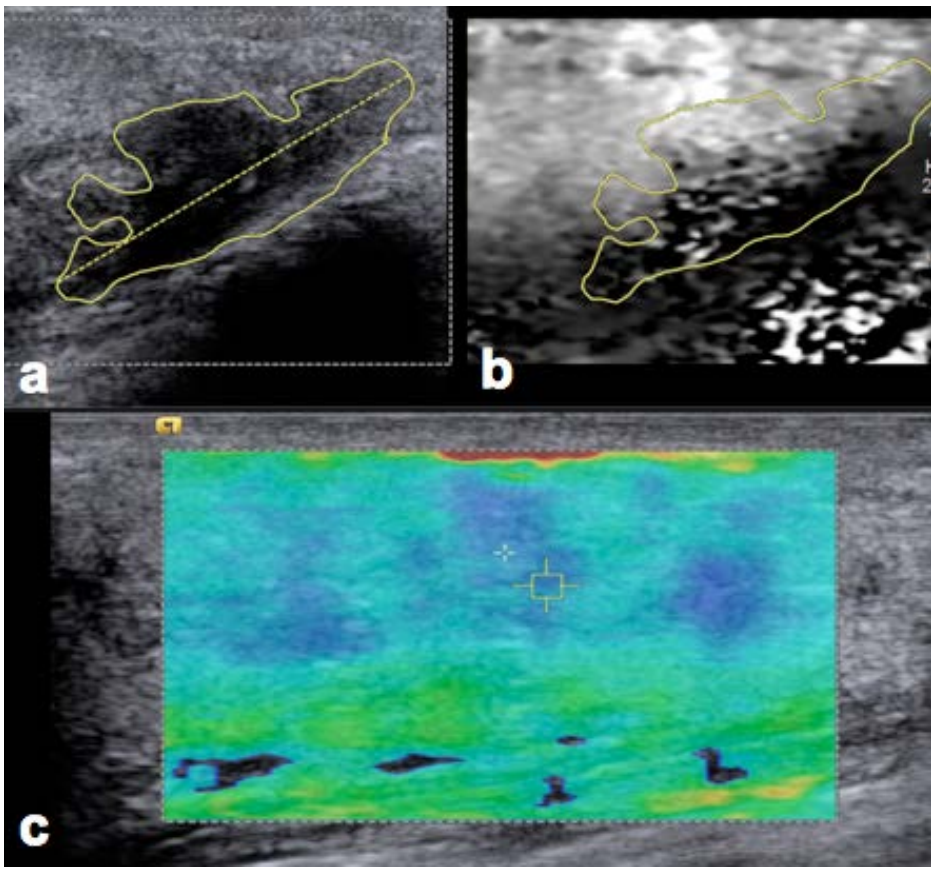

Figure 4. 34 years old woman. In physical examination right upper lateral quadrant described as mild tough and sensitive. (a) spacious, demarcated, lobulated contoured hypoechogenity field watched on ultrasound; (b) this area viewed softer than the tissue and formed hyperechoic color scattering on ARFI displacement map; (c) but any difference was not detected between normal and very hypoechogen visible area on VTIQ map (benign fibrocystic change). 


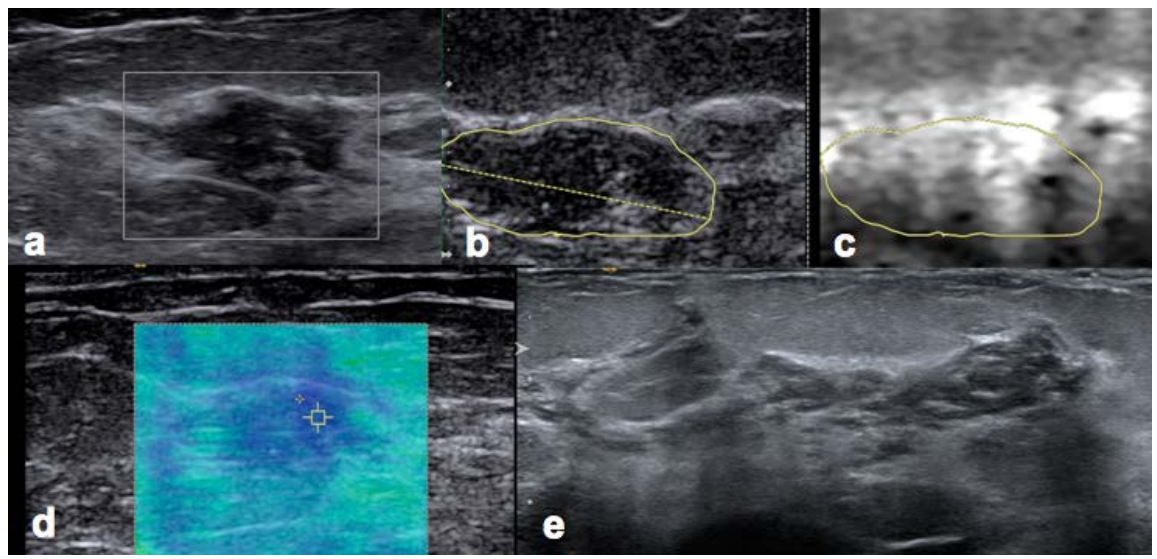

Figure 5. 36 year-old asymptomathic woman, hardness found during manual self examination. (a) hypoechogen, irregular contoured and internal septas on gray scale ultrasound. Degenerated fibroadenoma doubt has occurred; (b)-(c) more echogenic (soft) properties than ground tissue on ARFI displacement map; (d) similar homogen color pattern with the floor on the color VTIQ map; (e) After the second menstrual cycle control, lost the demarcation by showing more shape changes (compatible with focal fibrocystic complex).

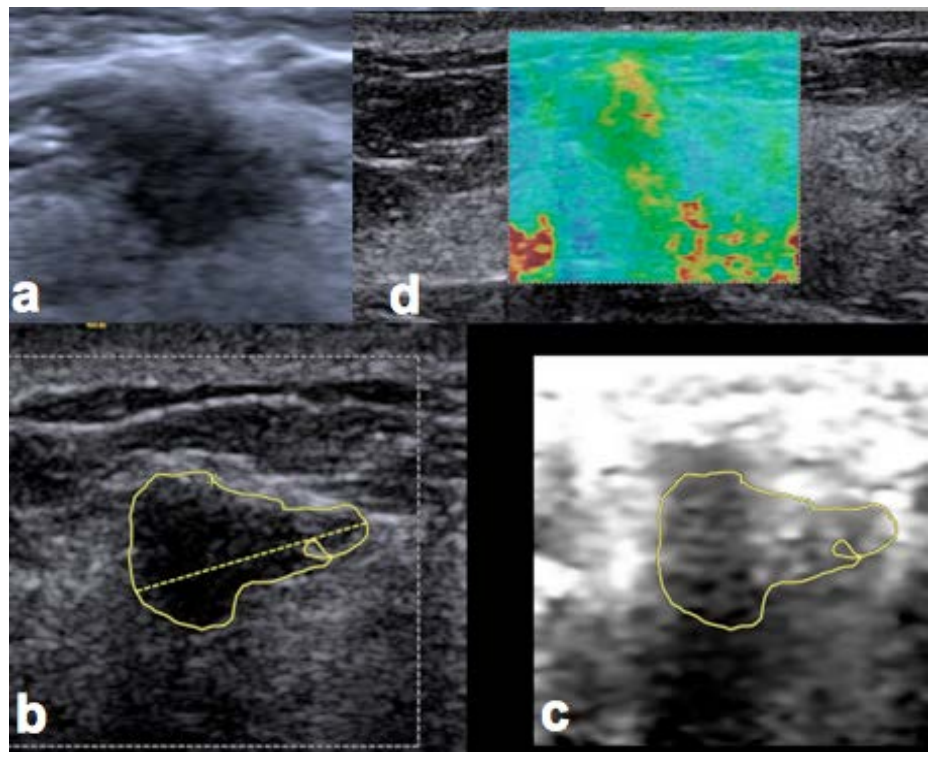

Figure 6. 39 year-old female patient referred with localized nodular palpable lesion at upper inner quadrant of the left breast. (a) macrolobulated contoured, homogeneous hypoechogen visualization of the lesion on gray scale US; (b)-(c) the inner contour of the lesion has softer inner structure on ARFI displacement map; (d) Although homogeneous hypoechogen content the color inside the lesion is variable on VTIQ examination. Degenerated fibroadenoma. In these types of lesions apparent dysmorphic due to degeneration, biopsy should be preferred to apply from the poles SWV maximally increased.

resolution colored SWV maps (as mentioned before, in our system it is called VTIQ) (Figure 7). With the new parameters imposed by elastography, the benign lesions such as degenerated fibroadenoma and papilloma which can mimic 
a malignant lesion, and the changes like fat necrosis and postoperative scar that concerning the operation area can be distinguished (Figure 8). In fact, before capturing the event called blind lesion in radiology, at the same time, after the identification of masses with malignant criteria, screening the other normal-looking quadrants can diagnose the iso echogenic DCIS, in some cases (Figure 9).

Furthermore, not only breast, but also for axillary lymph nodes, especially in the usual oval shape, SWV elastography has benefits in differential dignosis of benign or malignant lymphadenopathy with smooth-edged thin cortex and deterioration in the physiological hilar echo. Although there is not enough compatibility between studies about axillary and other lymphatic chains settled in the superficial lymph nodes, generally when cortical SWV values exceed the cut-off values, decision of biopsy can be given by evaluating also the other morphological findings together [39] [40] [41]. In our unit, for the lymph nodes which are thickened, nodularity gained or deleting the echo hilum of the cortex, SWV cut-off values are accepted meaningful $>2.4 \mathrm{~m} / \mathrm{sec}$, in terms of malignancy.

Overall, the first produced softwares of strain elastography which were extremely operator-dependent forms, difficult to adjust the manual compression degree are almost out of general clinical use. Adjusting the compression by graphical data and efforts of semiquantifying this system with color index or strain ratio measurements through color scales has led to relatively successful results, by means of the new RTE methods. However, after the spread of SWE method, tissue stiffness become to be measured more comfortably with lesser

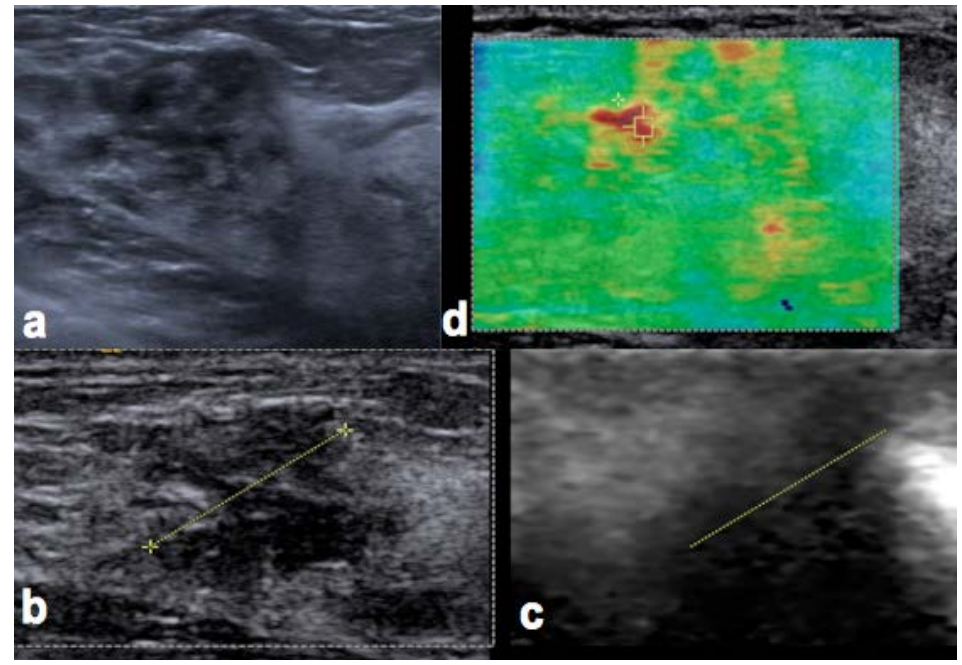

Figure 7. A 41 year-old case represented similar to these fibrocystic complexes with hardness palpated by herself at upper lateral quadrant of right breast. (a) heterogeneous hypoechogen, non-clear demarcated area on gray scale US; (b)-(c) much more rigid than the neighboring tissue and hypoechogen color scattering formation on ARFI displacement map; (d) intensive red-bright color transitions were associated with malignant SWV increases on VTIQ colored map. Tru-cut biopsy and histopathological diagnosis: High-grade DCIS. 


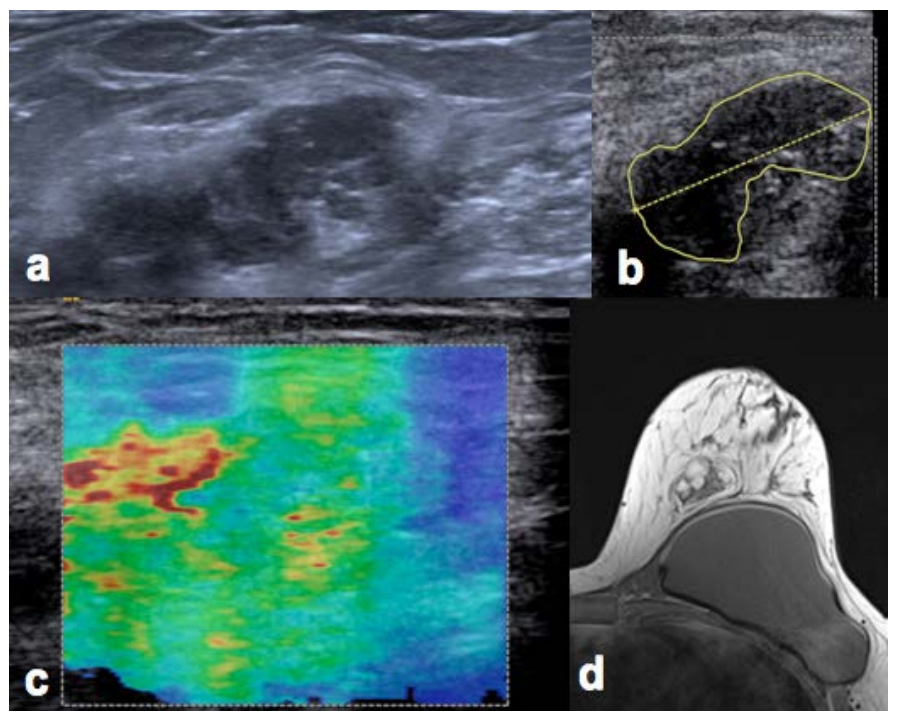

Figure 8. 47 years-old case with a history of a malignancy operation and therapy at lower-inner quadrant of left breast. (a) heterogeneous hypoechogen irregular contoured lesion on gray scale Usg; (b) tougher internal structure than the ground tissue on ARFI displacement map; (c) despite increased speeds of up to malign level on VTIQ $(3.4 \mathrm{~m} / \mathrm{s})$, because of lacking of a prominent desmoplastic halo and absence of expansion image around the lesion on elasto maps, malignancy was not considered initially. In addition, if looked at more carefully to gray scale images of this lesion which shows echogenic fat components inside; (d) in the MRI obtained on the same day, the oil cysts and changes at the borderline in T1a weighted axial images symbolizes the late postoperative mature fat necrosis.

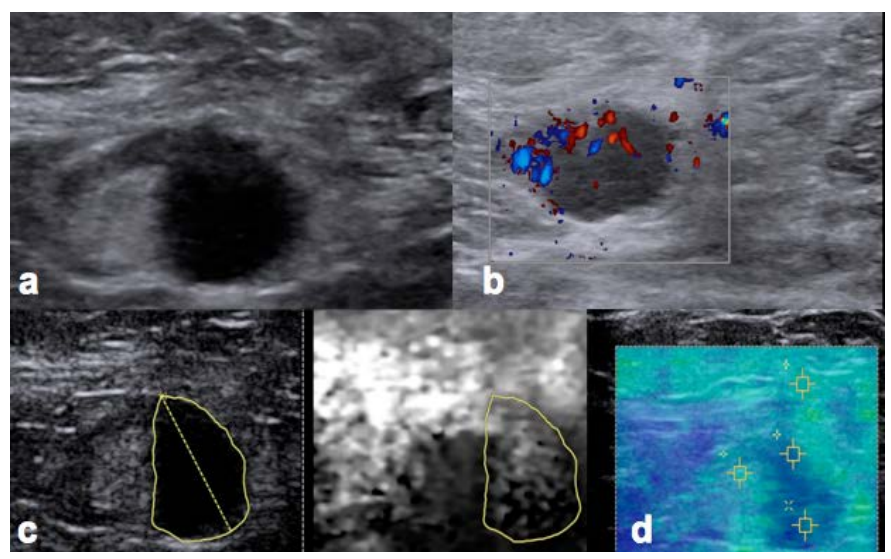

Figure 9. 54 years-old female patient whose mammography and breast USG previos year was described normal, but at this control; (a) on gray scale window; hypoechogen eccentric nodular thickening of advanced lymph node; (b) heterogeneous peripheral blood supply growth on doppler USG; (c)-1-2) soft inner structure on the ARFI displacement map; (d) coded with light green, light tougher to surrounding axillary fat tissue but the speed limit spectrum at benign level $(<2.4 \mathrm{~m} / \mathrm{s})$ on VTIQ map. Tru-cut biopsy: Benign reactive hyperplastic lymph node. 
operator dependent, quantitative techniques. The stiffness values of SWV measurements are defined as $\mathrm{m} / \mathrm{s}$ with the most commonly used ARFI system and as $\mathrm{kPa}$ with another one. Even though various and gradually renewed versions such as point shear wave, 2-D shear wave, 3-D shear wave; calculation of the microanatomical comppression deggree of tissue by a push-pulse mechanizm and measuring the shear-wave velocity during this push is the valid mechanism [42]. In malignant tissues, tighter cell groups and surrounding desmoplastic reaction leads to increase SWV, and at sizes exceeding a threshold value it is easier to recognize a lesion with benign morphology, such as mucinous carcinoma [43]. On the other hand, recognition of premalignant lesions like ductal carcinoma in-situ (DCIS) and to distinguish the solid lesions from complicated hypoechoic cysts are also facilitated [43] [44]. Therefore, because of the potential to reduce the false positive and/or false negative ultrasound evaluation rates of this method, a more accurate classification of various lesions in BIRADS categorization is feasible.

There are some limitations with this technique. Although quantitative elasticity information obtained by SWE has improved diagnostic performance, false results have been reported in $6.4 \%-36.6 \%$ of cases, in which the imaging results did not correlate with the pathologic results [45]. Also not easily accessible for the moment, over time, it is specified to take its place among the standard equipment of manufacturers. Utilization principles may be adopted by the appropriate application and short-term experiences. However, it does not have a complicated algorithm and difficult learning curve in terms of different tissues have different characteristics. Overall, though the background resolution persists lower than normal gray scale floor, new developments day by day on this issue are carried out by hardware and software upgrades that are used, currently. Although the activity decreases in deep-seated lesions, generally beyond very large breasts there are not any problem in breast. On the other hand, because of the artifacts caused by reflected waves the activity reduces in the superficial lesions very closed to skin. This problem can be circumvented easily with a suitable gel-pad support. In addition, though various companies use different terminology but the general principles are the same, these small differences can be solved easily by the help of the manufacturer or literature support. It has shown in the literature that VTIQ is a highly reliable method concerning intra- and inter-examiner agreement.

\section{Conclusion}

Strain elastography, which has numerous publications about in the radiology literature, could go a little step further albeit small with the new software supports, but probably due to manual compression and being very dependent on operator experience it has not found many places in the clinical-surgical practice. However, since it is cheaper and more accessible is still used especially systems with semiquantitative software. SWE, starting from a simple speed mea- 
surement, with color maps obtained after a moderate precompression and alternative 2D-3D developed softwares, just as Doppler, seems to be another aide to the gray scale sonography.

\section{Conflicts of Interest}

The authors have no conflict of interests to disclose.

\section{References}

[1] Goddi, A., Bonardi, M. and Alessi, S. (2012) Breast Elastography: A Literature Review. Journal of Ultrasound, 15, 192-198. https://doi.org/10.1016/j.jus.2012.06.009

[2] Stijven, S., Gielen, E., Bevernage, C., Horvath, M. and Meylaerts, L. (2013) Magnetic Resonance Imaging: Value of Diffusion-Weighted Imaging in Differentiating Benign from Malignant Breast Lesions. European Journal of Obstetrics \& Gynecology \& Reproductive Biology, 166, 215-220. https://doi.org/10.1016/j.ejogrb.2012.10.029

[3] Uematsu, T. (2014) Ultrasonographic Findings of Missed Breast Cancer: Pitfalls and Pearls. Breast Cancer, 21, 10-19. https://doi.org/10.1007/s12282-013-0498-7

[4] Tozaki, M. and Fukuma, E. (2011) Does Power Doppler Ultrasonography Improve the BI-RADS Category Assessment and Diagnostic Accuracy of Solid Breast Lesions? Acta Radiologica, 52, 706-710. https://doi.org/10.1258\%2Far.2011.110039

[5] Heng, H.G. and Widmer, W.R. (2010) Appearance of Common Ultrasound Artifacts in Conventional vs. Spatial Compound Imaging. Veterinary Radiology \& UItrasound, 51, 621-627. https://doi.org/10.1111/j.1740-8261.2010.01724.x

[6] Yildirim, D., Gurses, B., Eksci, B. and Kaur, A. (2011) Power Doppler Vocal Fremitus Breast Sonography: Differential Diagnosis with a New Classification Scheme-Power Doppler Vocal Fremitus Examination of Breast Lesions. Journal of Cancer Therapy, 2, 243-252. https://doi.org/10.4236/jct.2011.22031

[7] Barr, R.G. (2019) Future of Breast Elastography. Ultrasonography, 38, 93-105. https://doi.org/10.14366/usg.18053

[8] Dighe, M., Luo, S., Cuevas, C. and Kim, Y. (2013) Efficacy of Thyroid Ultrasound Elastography in Differential Diagnosis of Small Thyroid Nodules. European Journal of Radiology, 82, 274-280. https://doi.org/10.1016/j.ejrad.2013.01.009

[9] Dietrich, C.F., Barr, R.G., Farrokh, A., Dighe, M., Hocke, M., Christian Jenssen, C., et al. (2017) Strain Elastography-How To Do It? Ultrasound International Open, 3, E137-E149. https://doi.org/10.1055/s-0043-119412

[10] Youk, J.H., Gweon, H.M. and Son, E.J. (2017) Shear-Wave Elastography in Breast Ultrasonography: The State of the Art. Ultrasonography, 36, 300-309. https://doi.org/10.14366/usg.17024

[11] Nightingale, K., Soo, M.S., Nightingale, R. and Trahey, G. (2002) Acoustic Radiation Force Impulse Imaging: In Vivo Demonstration of Clinical Feasibility. Ultrasound in Medicine \& Biology, 28, 227-235. https://doi.org/10.1016/S0301-5629(01)00499-9

[12] Yang, H., Xu, Y., Zhao, Y., Yin, J., Chen, Z. and Huang, P. (2020) The Role of Tissue Elasticity in the Differential Diagnosis of Benign and Malignant Breast Lesions Using Shear Wave Elastography. BMC Cancer, 20, Article No. 930. https://doi.org/10.1186/s12885-020-07423-X

[13] Gong, X., Xu, Q., Xu, Z., Xiong, P., Yan, W. and Chen, Y. (2011) Real-Time Elastography for the Differentiation of Benign and Malignant Breast Lesions: A Meta-Analysis. Breast Cancer Research and Treatment, 130, Article No. 18. 
https://doi.org/10.1007/s10549-011-1745-2

[14] Frey, H. (2003) Realtime-Elastographie. A New Ultrasound Procedure for the Reconstruction of Tissue Elasticity. Der Radiologe, 43, 850-855.

https://doi.org/10.1007/s00117-003-0943-2

[15] Xiao, Y., Yu, Y., Niu, L., Qian, M., Deng, Z., Qiu, W. and Zheng, H. (2016) Quantitative Evaluation of Peripheral Tissue Elasticity for Ultrasound-Detected Breast Lesions. Clinical Radiology, 71, 896-904. https://doi.org/10.1016/j.crad.2016.06.104

[16] Shiina, T., Nightingale, K.R., Palmeri, M.L., Hall, T.J., Bamber, J.C., Barr, R.G., et al. (2015) WFUMB Guidelines and Recommendations for Clinical Use of Ultrasound Elastography: Part 1: Basic Principles and Terminology. Ultrasound in Medicine \& Biology, 41, 1126-1147. https://doi.org/10.1016/j.ultrasmedbio.2015.03.009

[17] Yildirim, D., Sahin, M., Tutar, O., Kayadibi, H., Kaur, A., Coskun, A.K. and Gumus, T. (2013) Ultrasound Elastography for the Differential Diagnosis of Nipple Retraction. Journal of Medical Ultrasonics, 40, 429-435.

https://doi.org/10.1007/s10396-013-0439-2

[18] Barr, R.G., Nakashima, K., Amy, D., Cosgrove, D., Farrokh, A., Schafer, F., et al. (2015) WFUMB Guidelines and Recommendations for Clinical Use of Ultrasound Elastography: Part 2: Breast. Ultrasound in Medicine \& Biology, 41, 1148-1160. https://doi.org/10.1016/j.ultrasmedbio.2015.03.008

[19] Zhou, J., Zhou, C., Zhan, W., Jia, X., Dong, Y. and Yang, Z. (2014) Elastography Ultrasound for Breast Lesions: Fat-to-Lesion Strain Ratio vs Gland-to-Lesion Strain Ratio. European Radiology, 24, 3171-3177. https://doi.org/10.1007/s00330-014-3366-8

[20] Song, G., Jing, L., Yan, M., Cong, S. and Wang, X. (2015) Influence of Various Breast Factors on the Quality of Strain Elastograms. Journal of Ultrasound in Medicine, 34, 395-400. https://doi.org/10.7863/ultra.34.3.395

[21] Sarvazyan, A.P., Rudenko, O.V., Swanson, S.D., Brian Fowlkes, J. and Emelianov, S.Y (1998) Shear Wave Elasticity Imaging: A New Ultrasonic Technology of Medical Diagnostics. Ultrasound in Medicine \& Biology, 24, 1419-1435. https://doi.org/10.1016/S0301-5629(98)00110-0

[22] Jayaraman, J., Indiran, V., Kannan, K. and Maduraimuthu, P. (2017) Acoustic Radiation Force Impulse Imaging in Benign and Malignant Breast Lesions. Cureus, 9, e1301. https://doi.org/10.7759/cureus.1301

[23] Gennisson, J.L., Deffieux, T., Fink, M. and Tanter, M. (2013) Ultrasound Elastography: Principles and Techniques. Diagnostic and Interventional Imaging, 94, 487-495. https://doi.org/10.1016/j.diii.2013.01.022

[24] Golatta, M., Schweitzer-Martin, M., Harcos, A., Schott, S., Gomez, C., Stieber, A., et al. (2013) Normal Breast Tissue Stiffness Measured by a New Ultrasound Technique: Virtual Touch Tissue Imaging. European Journal of Radiology, 82, 676-679. https://doi.org/10.1016/j.ejrad.2013.06.029

[25] Kim, Y.S., Park, J.G., Kim, B.S., Lee, C.H. and Ryu, D.W. (2014) Diagnostic Value of Elastography Using Acoustic Radiation Force Impulse Imaging and Strain Ratio for Breast Tumors. Journal of Breast Cancer, 17, 76-82. https://doi.org/10.4048/jbc.2014.17.1.76

[26] Ianculescu, V., Ciolovan, L.M., Dunant, A., Vielh, P., Mazouni, C., Delaloge, S., et al. (2014) Added Value of Virtual Touch IQ Shear Wave Elastography in the Ultrasound Assessment of Breast Lesions. European Journal of Radiology, 83, 773-777. https://doi.org/10.1016/j.ejrad.2014.01.021

[27] Youk, J.H., Gweon, H.M., Son, E.J., Kim, J.A. and Jeong, J. (2013) Shear-Wave 
Elastography of Invasive Breast Cancer: Correlation between Quantitative Mean Elasticity Value and Immunohistochemical Profile. Breast Cancer Research and Treatment, 138, 119-126. https://doi.org/10.1007/s10549-013-2407-3

[28] Evans, A., Whelehan, P., Thomson, K., McLean, D., Brauer, K., Purdie, C., et al. (2012) Invasive Breast Cancer: Relationship between Shear-Wave Elastographic Findings and Histologic Prognostic Factors. Radiology, 263, 673-677.

https://doi.org/10.1148/radiol.12111317

[29] Choi, W.J., Kim, H.H., Cha, J.H., Shin, H.J., Kim, H., Chae, E.Y., et al. (2014) Predicting Prognostic Factors of Breast Cancer Using Shear Wave Elastography. Ultrasound in Medicine \& Biology, 40, 269-274.

https://doi.org/10.1016/j.ultrasmedbio.2013.09.028

[30] Chang, J.M., Park, I.A., Lee, S.H., Kim, W.H., Bae, M.S., Koo, H.R., et al. (2013) Stiffness of Tumours Measured by Shear-Wave Elastography Correlated with Subtypes of Breast Cancer. European Radiology, 23, 2450-2458.

https://doi.org/10.1007/s00330-013-2866-2

[31] Wojcinski, S., Brandhorst, K., Sadigh, G., Hillemanns, P. and Degenhardt, F. (2013) Acoustic Radiation Force Impulse Imaging with Virtual Touch ${ }^{\mathrm{TM}}$ Tissue Quantification: Mean Shear Wave Velocity of Malignant and Benign Breast Masses. International Journal of Women's Health, 30, 619-627. https://doi.org/10.2147/IJWH.S50953

[32] Li, G., Li, D.W., Fang, Y.X., Song, Y.J., Deng, Z.J., Gao, J., et al. (2013) Performance of Shear Wave Elastography for Differentiation of Benign and Malignant Solid Breast Masses. PLoS ONE, 18, e76322. https://doi.org/10.1371/journal.pone.0076322

[33] Zhang, H., Shi, Q., Gu, J., Jiang, L., Bai, M., Liu, L., et al. (2014) Combined Value of Virtual Touch Tissue Quantification and Conventional Sonographic Features for Differentiating Benign and Malignant Thyroid Nodules Smaller than 10mm. Journal of Ultrasound in Medicine, 33, 257-264. https://doi.org/10.7863/ultra.33.2.257

[34] Gu, J., Du, L., Bai, M., Chen, H., Jia, X., Zhao, J. and Zhang, X. (2012) Preliminary Study on the Diagnostic Value of Acoustic Radiation force Impulse Technology for Differentiating between Benign and Malignant Thyroid Nodules. Journal of Ultrasound in Medicine, 31, 763-771. https://doi.org/10.7863/jum.2012.31.5.763

[35] Bai, M., Du, L., Gu, J., Li, F. and Jia, X. (2012) Virtual Touch Tissue Quantification Using Acoustic Radiation Force Impulse Technology: Initial Clinical Experience with Solid Breast Masses. Journal of Ultrasound in Medicine, 31, 289-294. https://doi.org/10.7863/jum.2012.31.2.289

[36] Tozaki, M., Isobe, S. and Sakamoto, M. (2012) Combination of Elastography and Tissue Quantification Using the Acoustic Radiation Force Impulse (ARFI) Technology for Differential Diagnosis of Breast Masses. Japanese Journal of Radiology, 30, 659-670. https://doi.org/10.1007/s11604-012-0106-3

[37] Nightingale, K., McAleavey, S. and Trahey, G. (2003) Shear-Wave Generation Using Acoustic Radiation force: In Vivo and ex Vivo Results. Ultrasound in Medicine \& Biology, 29, 1715-1723. https://doi.org/10.1016/j.ultrasmedbio.2003.08.008

[38] Wojcinski, S., Brandhorst, K., Sadigh, G., Hillemanns, P. and Degenhardt, F. (2013) Acoustic Radiation Force Impuşse Imaging with Virtual Touch Tissue Quantification: Measurements of Normal Breast Tissue and Dependence on the Degree of Pre-Compression. Ultrasound in Medicine \& Biology, 39, 2226-2232. https://doi.org/10.1016/j.ultrasmedbio.2013.06.014

[39] Meng, W., Xing, P., Chen, Q. and Wu, C. (2013) Initial Experience of Acoustic 
Radiation Force Impulse Ultrasound Imaging of Cervical Lymph Nodes. European Journal of Radiology, 82, 1788-1792. https://doi.org/10.1016/j.ejrad.2013.05.039

[40] Fujiwara, T., Tomokuni, J., Iwanaga, K., Ooba, S. and Haji, T. (2013) Acoustic Radiation Force Impulse Imaging for Reactive and Malignant/Metastatic Cervical Lymph Nodes. Ultrasound in Medicine \& Biology, 39, 1178-1183.

https://doi.org/10.1016/j.ultrasmedbio.2013.02.001

[41] Li, J., Chen, M., Cao, C.L., Zhou, L.Q., Li, S.G., Ge, Z.K., et al. (2020) Diagnostic Performance of Acoustic Radiation Force Impulse Elastography for the Differentiation of Benign and Malignant Superficial Lymph Nodes: A Meta-Analysis. Journal of Ultrasound in Medicine, 39, 213-222. https://doi.org/10.1002/jum.15096

[42] Athanasiou, A., Latorre-Ossa, H., Criton, A., Tardivon, A., Gennisson, J.L. and Tanter, M. (2017) Feasibility of Imaging and Treatment Monitoring of Breast Lesions with Three-Dimensional Shear Wave Elastography. Ultraschall in der Medizin, 38, 51-59. https://doi.org/10.1055/s-0034-1398980

[43] Bidlek, M., Kovacs, E., Feher, K. and Godeny, M. (2015) New Opportunities in Imaging of Breast Cancer. Magyar Onkológia, 59, 44-55.

[44] Pu, H., Zhang, X.L., Xiang, L.H., Zhang, J.L., Xu, G., Liu, H., et al. (2019) The efficacy of Added Shear Wave Elastography (SWE) in Breast Screening for Women with Inconsistent Mammography and Conventional Ultrasounds (US). Clinical Hemorheology and Microcirculation, 71, 83-94. https://doi.org/10.3233/CH-180398

[45] Park, S.Y., Choi, J.S., Han, B.K., Ko, E.Y. and Ko, E.S. (2017) Shear Wave Elastography in the Diagnosis of Breast Non-Mass Lesions: factors Associated with False negative and False Positive Results. European Radiology, 27, 3788-3798.

https://doi.org/10.1007/s00330-017-4763-6 